\title{
Effect of YAPI silencing on esophageal cancer
}

\author{
Jia Zhao',2 \\ Xiangnan $\mathrm{Li}^{1,2}$ \\ Yang Yang ${ }^{1,2}$ \\ Dengyan Zhu' ${ }^{1,2}$ \\ Chunyang Zhang ${ }^{1,2}$ \\ Donglei Liu',2 \\ Kai Wu ${ }^{1,2}$ \\ Song Zhao',2 \\ 'Department of Thoracic Surgery, \\ The First Affiliated Hospital, \\ Zhengzhou University, ${ }^{2}$ Key Thoracic \\ Tumour Experimental Laboratory \\ of Zhengzhou, Zhengzhou, Henan, \\ People's Republic of China
}

This article was published in the following Dove Press journal:

OncoTargets and Therapy

26 May 2016

Number of times this article has been viewed

Background: YAP1, the nuclear effector of the Hippo pathway, has become an attractive target for treatment of malignancies and is a candidate oncogene in esophageal cancer (EC). We hypothesized that knockdown of YAP1 could suppress EC and could be used for targeted therapy. However, there are few reports of the effect of YAP1 knockdown in EC.

Materials and methods: Quantitative real-time polymerase chain reaction and Western blot assays were performed to determine the expression levels of YAP1 mRNA and protein in primary EC tissue samples, EC cell lines, and controls. Immunohistochemistry was also performed to detect YAP1 protein expression in primary EC tumor and matched nontumor control tissues. YAP1-knockdown cell lines were constructed using short-hairpin RNA, and MTT, flow cytometry, and transwell chamber assays were used to analyze the effect of YAP1 knockdown on EC cell proliferation, apoptosis, and invasion. In vivo tumor formation assays were used to investigate the antitumor effect of YAP1 knockdown.

Results: We found that YAP1 mRNA and protein were upregulated in EC and that YAP1 expression correlated significantly with metastasis and tumor stage. We also found that YAP1 knockdown repressed cell proliferation and invasion and promoted apoptosis of EC cell lines. In addition, animal experiments revealed that YAP1 knockdown suppressed the growth of esophageal tumors in vivo.

Conclusion: Collectively, these data confirm our hypothesis that YAP1 knockdown suppresses EC and suggest that YAP1 knockdown could be exploited in the targeted gene therapy of EC in the future.

Keywords: esophageal cancer, Hippo, YAP1 knockdown, animal experiments

\section{Introduction}

Esophageal cancer (EC) is one of the most frequent malignant tumors and ranks as the sixth-leading cause of cancer-related death worldwide; ${ }^{1}$ esophageal squamous-cell carcinoma is the fastest-growing type of EC. In the People's Republic of China, the incidence of EC has tended to decrease over the past 20 years; however, in the last 5 years, the incidence has increased, due to factors including environmental change. ${ }^{1}$ The prognosis of EC is poor, with a 5-year survival rate of $26.2 \%$ and a recurrence rate of $>40 \% .^{2-4}$ Therefore, there is an urgent need for novel therapeutic strategies to improve the survival rates of patients with EC. Targeted gene therapy, which is a relatively new paradigm in medicine with enormous therapeutic potential, is the most promising prospect for improvement in outcomes of EC. ${ }^{5}$ Hence, it is crucial to identify therapeutic targets for EC treatment.

It is generally accepted that the main reason for cancer-related death is complications arising from metastasis. ${ }^{6}$ Tumor metastasis is a complex process involving numerous transcription factors and pathways, ${ }^{6}$ which represent potentially tractable therapeutic targets. Pathways that regulate transcription in response to extracellular cues are of particular interest. The Hippo (Hpo) pathway is one such pathway that
Correspondence: Song Zhao

Department of Thoracic Surgery, The First Affiliated Hospital of Zhengzhou University, No I Jianshe East Road, Zhengzhou 450052, Henan, People's Republic of China

Tel +86 37| 6796 7I52

Email songzhaozz@।63.com
OncoTargets and Therapy 2016:9 3/37-3/46 Dovepress ff in 0 http://dx.doi.org/10.2147/0TTS 102338 (c) (1) (5) 2016 hhao et al. This work is published and licensed by Dove Medical Press Limited. The full terms of this license are available at https://www.dovepress.com/terms.php cC ${ }_{\mathrm{BY}} \mathrm{NC}$ and incorporate the Creative Commons Attribution - Non Commercial (unported, v3.0) License (http://creativecommons.org/licenses/by-nc/3.0/). By accessing the work you hereby accept the Terms. Non-commercial uses of the work are permitted without any further permission from Dove Medical Press Limited, provided the work is properly attributed. For permission for commercial use of this work, please see paragraphs 4.2 and 5 of our Terms (https://www.dovepress.com/terms.php). 
acts to regulate gene expression in response to changes in cell shape, density, and adhesion..$^{7-11}$

The Hpo pathway is a major regulator of organ size and the balance between cell proliferation and apoptosis. ${ }^{12,13}$ YAP1 as one component of the Hpo pathway in mammals is the ortholog of Yorkie (Yki) in Drosophila, which is the downstream effector of the Hpo pathway, and it negatively regulates the Hpo pathway. Many recent studies have documented oncogenic effects of YAP1. In particular, YAP1 amplification and overexpression are found in various human cancers, including pancreatic cancer, breast cancer, hepatocellular carcinoma, medulloblastoma, esophageal squamouscell carcinoma, and oral squamous-cell carcinomas. ${ }^{6,14-18}$ Overholtzer et $\mathrm{al}^{19}$ reported that overexpression of YAP1 promoted epithelial-mesenchymal transition, growth factorindependent proliferation, and anchorage-independent growth and repressed apoptosis in the nontransformed mammary epithelial cell line MCF10A. In addition, amplification and overexpression of YAP1 are observed in various mouse models of cancer. ${ }^{7,15,20}$ However, YAP1 has also been widely reported as a tumor suppressor. For example, YAP1 increases the ability of $\mathrm{p} 73$ to induce apoptosis during cisplatin-induced DNA damage. ${ }^{21}$ Basu et al reported that Akt phosphorylates YAP1, resulting in its inactivation, and that this leads to repression of the proapoptotic gene expression response following cellular damage. ${ }^{22}$ Similarly, Danovi et $\mathrm{al}^{23}$ also reported that YAP1 is a critical mediator of c-Jun-dependent apoptosis. In addition, the expression level of YAP1 was significantly downregulated in a subset of breast cancers, and knockdown promotes migration and invasion of breast cancer cells. $^{24}$

Considering all the above evidence, it seems likely that YAP1 may have a dual role in signaling, since it is located at the intersection of many molecular pathways, and its role may therefore depend on context, stress, and/or cell lineage. ${ }^{25}$ Muramatsu et al reported that YAP1 is a candidate oncogene for esophageal squamous-cell carcinoma in EC. ${ }^{18}$ Therefore, we hypothesized that YAP1 knockdown might suppress EC; however, there are few reports of the effect of YAP1 knockdown in EC.

In the present study, we investigated the effect of YAP1 knockdown in EC. We determined the expression of YAP1 mRNA and protein in samples from primary EC cases and cell lines by quantitative real-time polymerase chain reaction (qRT-PCR) and Western blot. Associations between YAP1 expression and clinicopathological characteristics of EC were also explored. The effects of YAP1 knockdown on proliferation, apoptosis, and invasion of EC cell lines were also investigated, and we performed mouse xenograft experiments to explore the effect of YAP1 knockdown on the growth of esophageal tumors in vivo. The results suggest a novel potential therapeutic target for EC.

\section{Materials and methods Clinical samples}

This study was approved by the Ethics Committee of The First Affiliated Hospital, Zhengzhou University, and every patient provided written informed consent. None of the patients included underwent any treatment prior to surgery or had synchronous or metachronous multiple cancers in other organs.

EC samples and adjacent normal esophageal tissues were obtained from 50 patients with esophageal squamous-cell carcinoma who underwent routine surgery in the Department of Thoracic Surgery, The First Affiliated Hospital, Zhengzhou University, in 2014. All samples were snap frozen in liquid nitrogen and then stored at $-70^{\circ} \mathrm{C}$.

In addition, the clinical data of 72 patients with esophageal squamous-cell carcinoma were analyzed retrospectively. The disease stage was defined according to the International Union against Cancer and following the pathological tumor, node, metastasis (pTNM) classification. The clinical data presented in Table 1 were available for all 72 patients.

Table I Correlation between clinicopathological characteristics and expression of YAPI in the EC patients $(\mathrm{N}=72)$

\begin{tabular}{llllll}
\hline Characteristics & $\mathbf{n}$ & YAPI+ & Percent & $\chi^{2}$ & P-value \\
\hline Age, years & & & & & \\
$\quad<60$ & 39 & 27 & 69.2 & 0.106 & 0.745 \\
$\quad \geq 60$ & 33 & 24 & 72.7 & & \\
Sex & & & & & \\
$\quad$ Male & 57 & 41 & 72.0 & 0.159 & 0.690 \\
$\quad$ Female & 15 & 10 & 66.7 & & \\
Tumor location & & & & & \\
$\quad$ Upper esophagus & 12 & 9 & 75.0 & 0.153 & 0.926 \\
$\quad$ Middle esophagus & 39 & 27 & 69.2 & & \\
$\quad$ Lower esophagus & 21 & 15 & 71.4 & & \\
Differential grade & & & & & \\
$\quad$ Well & 18 & 12 & 66.7 & 0.320 & 0.852 \\
$\quad$ Middle & 34 & 24 & 70.6 & & \\
$\quad$ Poor & 20 & 15 & 75.0 & & \\
LN metastasis & & & & & \\
$\quad$ Negative & 46 & 28 & 60.9 & 6.121 & 0.013 \\
$\quad$ Positive & 26 & 23 & 88.4 & & \\
PTNM stage & & & & & \\
I & 14 & 7 & 50.0 & 7.488 & 0.024 \\
II & 34 & 23 & 67.6 & & \\
III & 24 & 21 & 87.5 & & \\
\hline
\end{tabular}

Abbreviations: EC, esophageal cancer; LN, lymph node; PTNM, pathological tumor, node, metastasis. 


\section{Immunohistochemistry}

Tissues were fixed with $4 \%$ paraformaldehyde and embedded in paraffin blocks. Tissue sections $(4 \mu \mathrm{m})$ were processed for immunohistochemical examination. YAP1 protein expression in tumor, and adjacent normal esophageal, tissues was determined by the horseradish peroxidase immunohistochemical method, using an anti-YAP1 antibody (1:100; GeneTex, Inc., San Antonio, CA, USA), according to the manufacturer's instructions. For evaluation of YAP1 expression, staining intensity was scored as 0 (negative), 1 (weak), 2 (medium), or 3 (strong). The extent of staining was scored as $0(0 \%)$, $1(1 \%-25 \%), 2(26 \%-50 \%), 3(51 \%-75 \%)$, or $4(76 \%-$ $100 \%$ ), according to the percentage of positively stained areas in relation to the whole tissue sample area. The final staining score (0-7) was the sum of the staining extent and intensity scores. The samples were assessed by two experienced pathologists who were blinded to the patients' details.

\section{Cell lines and transfection}

Two esophageal squamous-cell carcinoma cell lines, EC109 and TE10, were purchased from American Type Culture Collection (Manassas, VA, USA), and human esophageal epithelial cells were obtained from Sciencell Research Laboratories (Carlsbad, CA, USA). The cells were grown in the Roswell Park Memorial Institute 1640 medium (HyClone, Logan, UT, USA) containing 10\% fetal bovine serum (HyClone), $100 \mu \mathrm{g} / \mathrm{mL}$ of streptomycin, and $100 \mathrm{IU} / \mathrm{mL}$ of penicillin in a $5 \% \mathrm{CO}_{2}$ humidified incubator at $37^{\circ} \mathrm{C}$.

For YAP1-knockdown experiments, a short-hairpin RNA (shRNA) expression vector targeting YAP1 (sh-YAP1; SH-012200-02-10; Thermo Fisher Scientific, Waltham, MA, USA) was used, while a nontargeting sequence (shOT; s-004000-02; Thermo Fisher Scientific) was used as a negative control. Cells were infected with an multiplicity of infection of 5-10 in the presence of polybrene $(4 \mu \mathrm{g} / \mathrm{mL}$; Sigma-Aldrich Co., St Louis, MO, USA) and were selected using puromycin (Sigma-Aldrich Co.) after 72 hours of infection. Then, bulk cell populations were collected and screened for YAP1 expression.

\section{Western blot analysis}

Total proteins were extracted from frozen EC and adjacent normal tissues or cultured cells using radioimmunoprecipitation assay buffer (Beyotime, Shanghai, People's Republic of China), separated by sodium dodecyl sulfate polyacrylamide gel electrophoresis, and transferred to Immobilon Hybond-C membranes (Thermo Fisher Scientific) with a tank-based blotting system. Then, immunological examinations were performed according to the standard techniques.
Primary antibodies against YAP1 (GeneTex, Inc.), Ki67 (Genmed Scientifics, Inc., Arlington, MA, USA), Bcl-2 (Abcam, Cambridge, UK), Bax (Abcam), MMP-2 (SigmaAldrich Co.), MMP-9 (Sigma-Aldrich Co.), and GAPDH (Genmed Scientifics, Inc.) and peroxidase-conjugated antiIgG secondary antibodies (Abcam) were used in the Western blot analysis. Signals were visualized using the Pierce ECL Substrate Western blot detection system (Thermo Fisher Scientific) and the Molecular Imager ChemiDoc XRS System (Bio-Rad Laboratories Inc., Hercules, CA, USA). The integrated density of protein bands was quantified using Image software (Version 1.6; National Institutes of Health, Bethesda, MD, USA).

\section{qRT-PCR}

Total RNA samples were extracted from frozen EC and adjacent normal tissues or cultured cells using the TRIzol reagent (Thermo Fisher Scientific), according to the manufacturer's instructions, and reverse transcribed into complementary DNA. The expression of YAP1 mRNA was detected by SYBR Green RT-PCR, and the expression of $G A P D H$ was used for normalization. RT-PCR was carried out on an ABI 7500 Fast Real-Time PCR System (Thermo Fisher Scientific).

\section{MTT assay}

Cell suspensions of $3 \times 10^{4}$ cells/well were seeded in 96-well plates and incubated overnight in a humidified incubator at $37^{\circ} \mathrm{C}, 5 \% \mathrm{CO}_{2}$ Cell growth was monitored every day for a period of 4 days. Next, $20 \mu \mathrm{L}$ of MTT $(5 \mathrm{mg} / \mathrm{mL}$, SigmaAldrich Co.) was added to each well, followed by further incubation for 4 hours. Then, culture medium was removed from the wells and $150 \mu \mathrm{L}$ of dimethyl sulfoxide (SigmaAldrich Co.) was added to dissolve the purple MTT-formazan crystals. The absorbance of each well was read at $570 \mathrm{~nm}$ using a microplate reader (Thermo Fisher Scientific).

\section{Flow cytometry analysis of apoptosis}

Exponential-phase cells $\left(2 \times 10^{5}\right)$ were collected, washed with phosphate-buffered saline, resuspended in $100 \mu \mathrm{L}$ binding buffer, and stained with $2.5 \mu \mathrm{L}$ Annexin V-fluorescein isothiocyanate and $5 \mu \mathrm{L}$ propidium iodide (PI) at room temperature for 10 minutes (BioVision, Milpitas, CA, USA). Then, fluorescence of fluorescein isothiocyanate and PI was detected by flow cytometry.

\section{Cell invasion assay}

Cell invasion assays were carried out using transwell chambers. Cells $\left(2 \times 10^{4}\right)$ were added into the top chambers 
that were coated with the Matrigel membrane (24-well insert, $8 \mu \mathrm{m}$ pore size; BD Biosciences, San Jose, MA, USA). Top chambers contained serum-free medium, and full medium supplemented with $10 \%$ fetal bovine serum was added to the bottom chamber as a chemoattractant. Following incubation of the cells for 24 hours at $37^{\circ} \mathrm{C}, 5 \% \mathrm{CO}_{2}$, the cells that did not invade through the pores were wiped out with a cotton swab. The cells on the lower surface of the membrane were immobilized in 4\% paraformaldehyde, stained in $0.5 \%$ crystal violet, rinsed in phosphate-buffered saline, and counted (five high-power fields per chamber) under an inverted microscope (Olympus Corporation, Tokyo, Japan).

\section{In vivo tumor formation assay}

Animal experiments were approved by the Institutional Review Board and carried out in compliance with the United Kingdom Coordinating Committee on Cancer Research guidelines for the welfare of experimental animals. For the in vivo study, EC109 sh-nontargeted and EC109 sh-YAP1 cells $\left(1 \times 10^{4}\right)$ were injected subcutaneously into the dorsal flanks of nude mice. Subcutaneous tumor sizes were measured weekly using manual calipers. Tumor volumes were calculated using the following formula ${ }^{26}$

$$
\text { Tumor volume }=\frac{\text { length } \times \text { width }^{2}}{2}
$$

Four weeks later, mice were sacrificed and tumor weights were measured. Moreover, the expression levels of Ki67, Bcl-2, Bax, MMP-2, and MMP-9 proteins in xenografts were detected by Western blot analysis.

\section{Statistical analyses}

Statistical analyses were performed using GraphPad Prism 5 (GraphPad Software, Inc., La Jolla, CA, USA). The results are presented as mean \pm standard deviation. Differences were analyzed by Student's $t$-test or chi-square test and were considered statistically significant at $P<0.05$.

\section{Results}

\section{YAPI mRNA and protein are overexpressed in EC}

The expression levels of YAP1 mRNA and protein in the tumor tissues and corresponding adjacent normal esophageal tissues of 50 EC patients were determined by qRT-PCR and Western blot. The results showed that the expression levels of both YAP1 mRNA and protein in the tumor tissues were significantly higher than those in the corresponding normal tissues (Figure 1A and B). Immunohistochemical examination was also performed and revealed that the esophageal tumor samples displayed clear, strong positive staining for YAP1 compared with the controls (Figure 1C and D). The expression levels of YAP1 mRNA and protein in two human EC cell lines (EC109 and TE10) were also determined, and the results demonstrated that the levels of both YAP1 mRNA and protein were significantly increased in EC109 and TE10 cell lines compared to those in human esophageal epithelial cells (Figure 1E and F).

In addition, we retrospectively analyzed the clinical data of 72 patients with EC and found a correlation between YAP1 expression and clinicopathological characteristics of EC (Table 1). No positive correlations with age, sex, tumor location, and differential grade were observed; however, there were significant correlations with lymph node metastasis $(P=0.013)$ and $p$ TNM stage $(P=0.024)$. The aberrant expression of YAP1 in EC tumor samples and cell lines suggests that it could play an important role in the pathogenesis of EC.

\section{Proliferation-inhibition effect of YAP I knockdown in EC cells}

To assess the effect of YAP1 on the proliferation of EC cells, endogenous YAP1 expression was modulated in EC109 and TE10 cell lines using an RNA interference strategy. YAP1 expression in control (nontargeting shRNA) and YAP1knockdown cells was detected by Western blot. As shown in Figure 2A and B, both EC109 and TE10 YAP1-knockdown cells had obvious decreases in YAP1 expression compared with controls. The proliferation of control and YAP1knockdown cells was analyzed by MTT assay. The results showed that both EC109 and TE10 YAP1-knockdown cells demonstrated dramatically decreased proliferation rates, compared with controls, indicating that YAP1 knockdown represses cell proliferation (Figure $2 \mathrm{C}$ and $\mathrm{E}$ ). In addition, we used Western blot to detect the expression levels of Ki67, a marker of tumor proliferation. The expression level of Ki67 in YAP1-knockdown EC109 and TE10 cells was significantly decreased compared to that in controls, providing further evidence that YAP1 knockdown represses cell proliferation (Figure 2D and F).

\section{Promotion of apoptosis by YAPI knockdown in EC cells}

The effect of YAP1 on the apoptosis of EC cells was determined using an Annexin/PI flow cytometry assay to analyze EC109, TE10 YAP1-knockdown, and control cells. The results demonstrated that both EC109 and TE10 YAP1-knockdown cells underwent significantly higher levels of apoptosis 
A

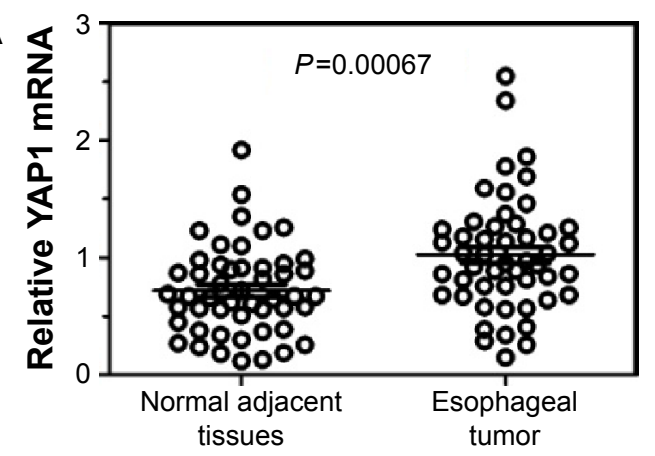

C

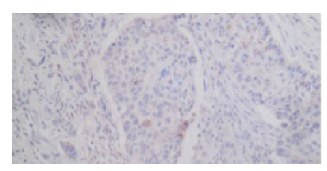

Normal adjacent to the tumor
Esophageal tumor

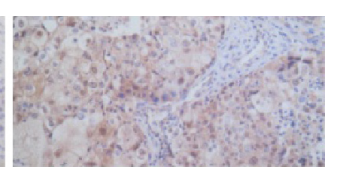

E

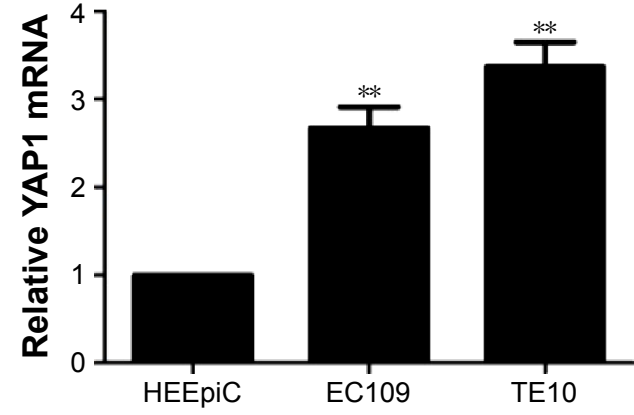

B

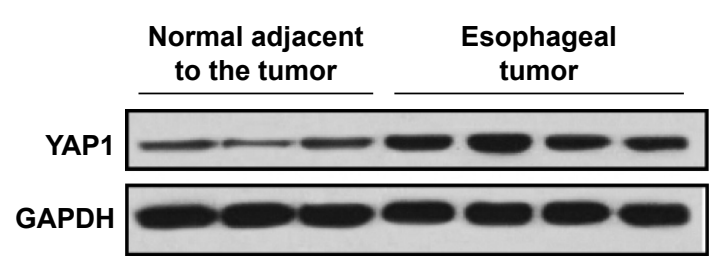

D

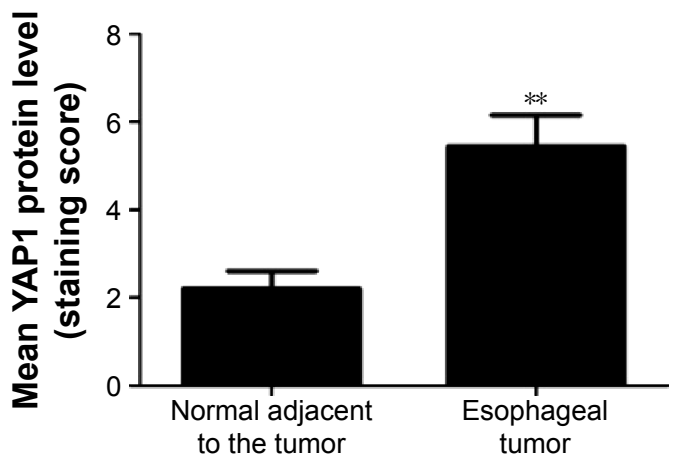

$\mathbf{F}$

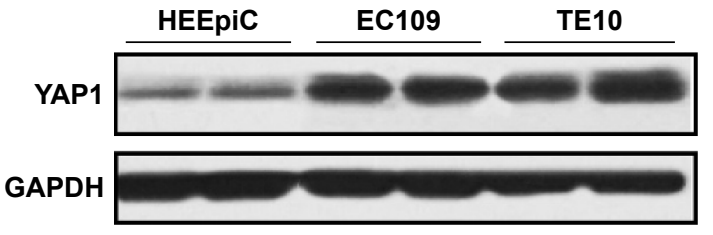

Figure I The expression levels of YAPI mRNA and protein in primary EC cases and cell lines.

Notes: (A and E) qRT-PCR shows that YAPI mRNA was significantly upregulated in 50 primary EC tissue samples and EC cell lines compared to nonmalignant control samples. (B and F) The expression level of YAPI protein was significantly increased in primary EC tissues and cell lines compared to nontumor controls. (C and $\mathbf{D}$ ) Esophageal tumor samples displayed clear, strong positive staining for YAPI compared with controls (original magnification $\times 400$ ). $* * P<0.01$.

Abbreviations: EC, esophageal cancer; qRT-PCR, quantitative real-time polymerase chain reaction; HEEpiC, human esophageal epithelial cells.

compared with controls (Figure 3A and C). Moreover, the expression levels of the apoptosis-related proteins Bcl-2 and Bax were detected by Western blot. The expression level of the antiapoptotic Bcl-2 protein in YAP1-knockdown EC109 and TE10 cells was significantly lower than that in the control group, while that of the proapoptotic Bax protein was increased (Figure 3B and D). These data indicate that YAP1 knockdown promotes apoptosis of EC cells.

\section{Inhibition of invasion by YAPI knockdown in EC cells}

To investigate the effect of YAP1 on invasion of EC cells, invasion assays with EC109 and TE10 YAP1-knockdown and control cells were performed using transwell chambers. As shown in Figure 4A and B, both EC109 and TE10 YAP1knockdown cells had significantly reduced invasion capacity in comparison with controls, indicating that YAP1 knockdown efficiently inhibits the invasion activity of EC cells. Furthermore, the expression levels of the markers of tumor invasion, MMP-2 and MMP-9, were examined by Western blot. The results showed that the expression levels of both MMP-2 and MMP-9 were significantly lower in EC109 and TE10 YAP1-knockdown cells than those in controls, (Figure 4C and D) further indicating that YAP1 knockdown inhibits the invasion activity of EC cells.

\section{YAPI knockdown suppresses the growth of esophageal tumors in vivo}

To explore the effect of YAP1 knockdown on the growth of esophageal tumors in vivo, YAP1-knockdown EC109 and non-targeted control cells $\left(1 \times 10^{4}\right)$ were subcutaneously injected into the dorsal flanks of nude mice. The volumes 
A

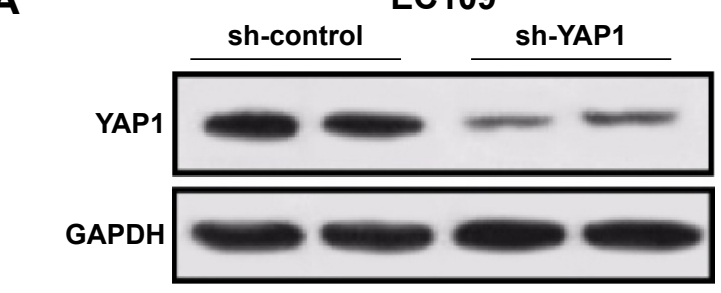

C

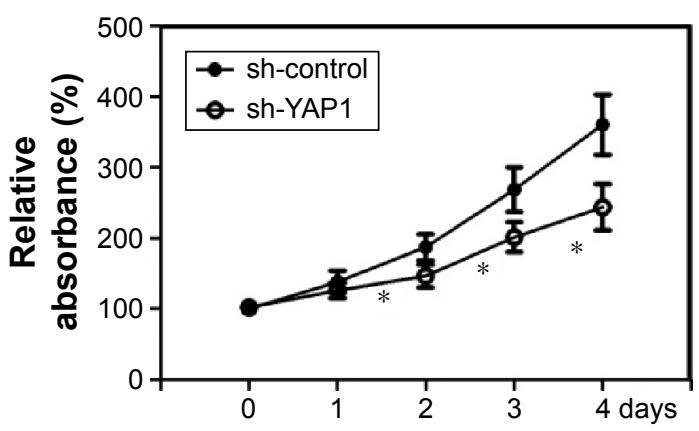

E

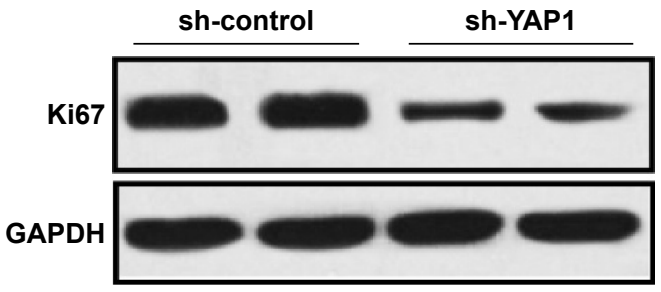

B

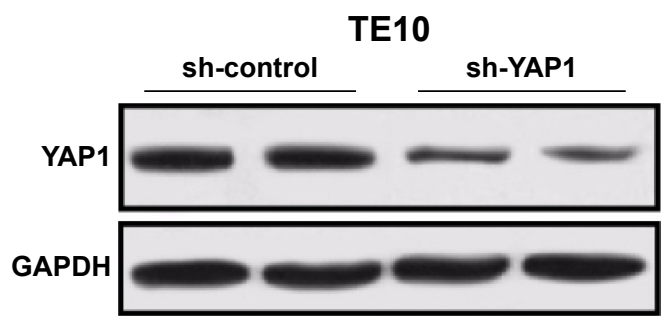

D

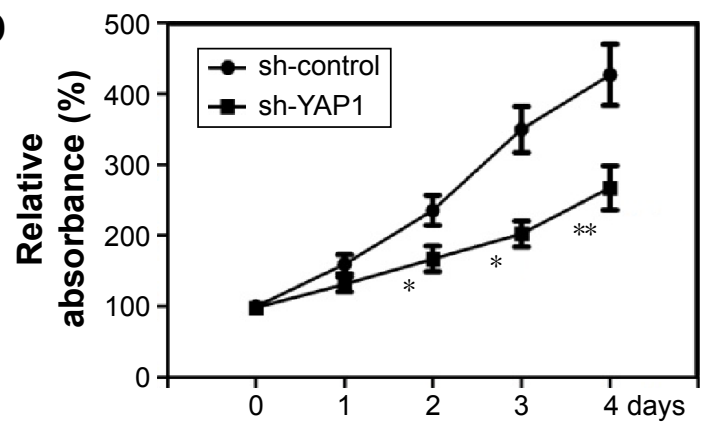

$\mathbf{F}$

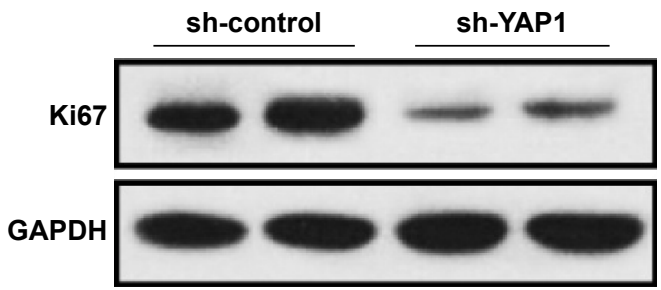

Figure 2 YAPI knockdown inhibited proliferation of EC cell lines.

Notes: (A and B) Both ECI09 and TEI0 YAPI-knockdown cells had clearly reduced YAPI expression compared with controls. (C and D) Both ECI09 and TEI0 YAPIknockdown cells (sh-YAPI) demonstrated dramatic decreases in proliferation rates compared with controls (sh-control). (E and $\mathbf{F}$ ) The expression levels of Ki67 in both ECI09 and TEI0 YAPI-knockdown cells (sh-YAPI) were significantly reduced compared to those in the control groups (sh-control). $* P<0.05$; $* * P<0.01$.

Abbreviations: EC, esophageal cancer; sh-YAPI, short-hairpin RNA expression vector targeting YAPI.

A

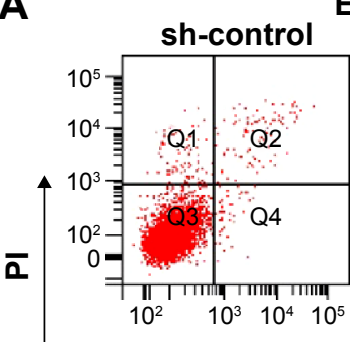

\section{EC109}

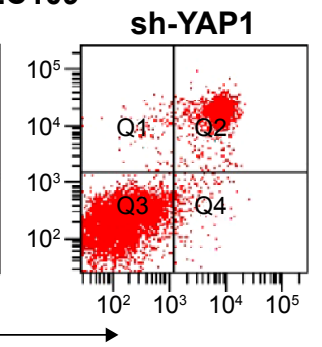

\section{Annexin V-FITC}

C

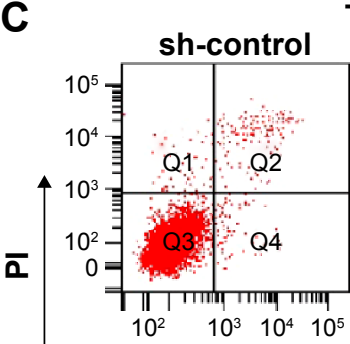

Annexin V-FITC
TE10

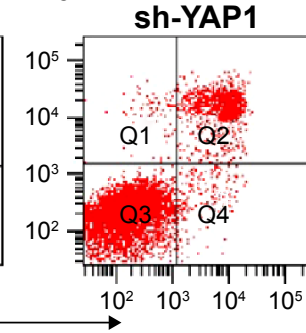

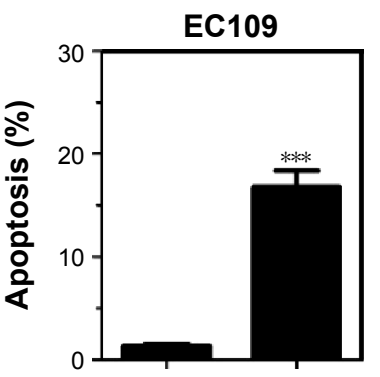

sh-control sh-YAP1

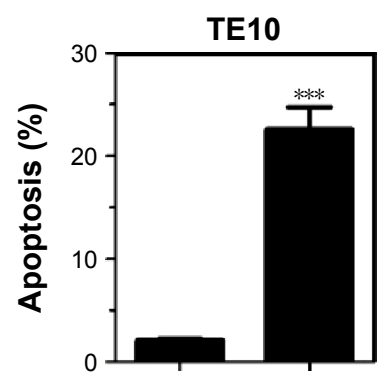

sh-control sh-YAP1

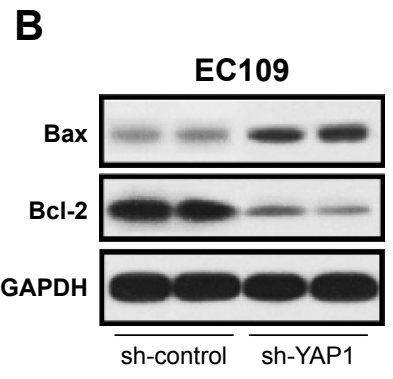

D

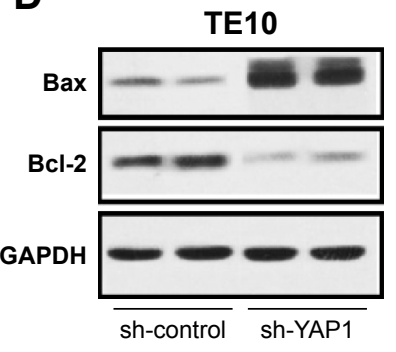

Figure 3 YAPI knockdown promoted apoptosis of EC cell lines.

Notes: (A and C) Both ECI09 and TEI0 YAPI-knockdown cells (sh-YAPI) had significantly increased apoptosis compared to controls (sh-control). (B and D) The expression levels of $\mathrm{Bcl}-2$ protein were significantly lower in YAPI-knockdown ECI09 and TEI0 cells (sh-YAPI) than those in controls (sh-control), while the expression levels of Bax protein were increased. *** $P<0.001$.

Abbreviations: EC, esophageal cancer; sh-YAPI, short-hairpin RNA expression vector targeting YAPI; PI, propidium iodide; FITC, fluorescein isothiocyanate. 

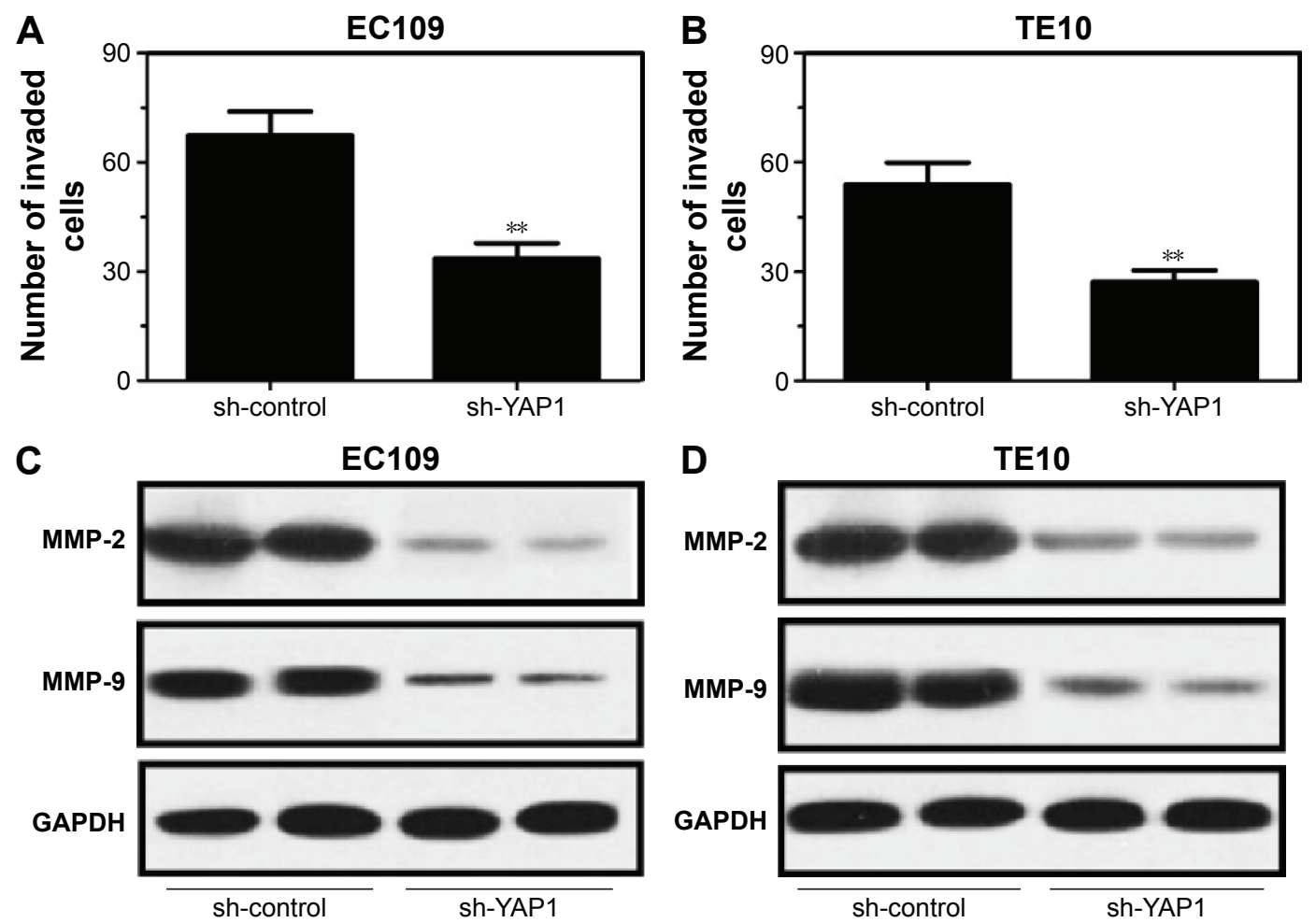

D

TE10

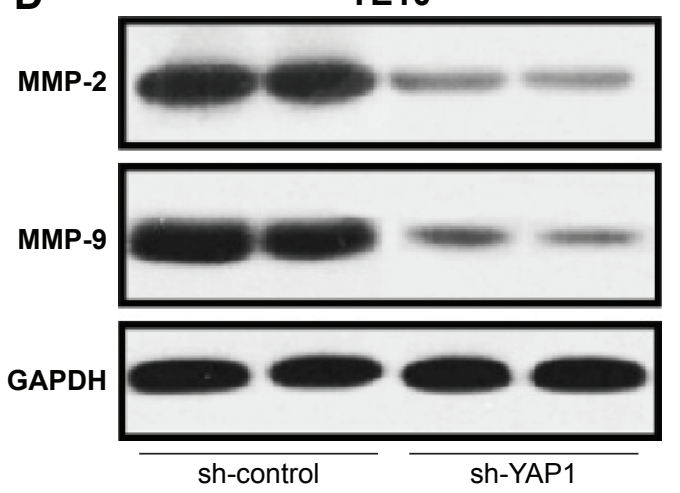

Figure 4 YAPI knockdown inhibited invasion by EC cell lines.

Notes: (A and C) ECI09 and TEIO YAPI-knockdown cells (sh-YAPI) had significantly lower invasion capacity compared to controls (sh-control). (B and D) The expression levels of both MMP-2 and MMP-9 proteins in YAPI-knockdown ECI09 and TEI0 cells (sh-YAPI) were significantly lower than those in controls (sh-control). **P $<0.01$.

Abbreviations: EC, esophageal cancer; sh-YAPI, short-hairpin RNA expression vector targeting YAPI.

of subcutaneous tumors were measured weekly, and the mean tumor volume in the YAP1-knockdown group was significantly reduced compared to that in the control group (Figure 5A). After 4 weeks, the mice were sacrificed and the tumors weighed. The mean tumor weight in the YAP1knockdown group was significantly lower than that in the control group (Figure 5B). Furthermore, the expression levels of Ki67, Bcl-2, Bax, MMP-2, and MMP-9 proteins in xenografts were examined by Western blot. As illustrated in Figure 5C, the expression levels of Ki67, Bax, MMP-2, and MMP-9 proteins were lower in the YAP1-knockdown group than those in the control group, while the expression level of Bcl-2 was higher in YAP1-knockdown tumor cells than that in the control group. These results indicate that YAP1 knockdown suppresses the growth of esophageal tumors in vivo.

\section{Discussion}

The components of the Hpo pathway were identified in Drosophila and include Warts protein kinase, Hpo kinase, Mats adaptor protein, Salvador adaptor protein, and Yki nuclear transcriptional coactivator. When the Hpo pathway is activated, Yki is dephosphorylated and retained in the cytoplasm. Mammalian Hpo pathway proteins are highly homologous to those in Drosophila. YAP1 is the mammalian ortholog of Drosophila Yki, the downstream effector and negative regulator of the Hpo pathway., ${ }^{7,28}$ Many roles have been reported for the Hpo pathway, including regulation of cell proliferation and apoptosis, and the pathway has been associated with malignancies. ${ }^{29-33}$ As the nuclear effector of the Hpo pathway, YAP1 is an attractive target molecule for the development of cancer therapies. Data from the literature suggest apparently conflicting roles for YAP1 in carcinogenesis; in that it has been reported to exhibit both oncogenic and tumor-suppressive properties in distinct in vivo and in vitro models. ${ }^{6,14-18,21-24}$ Muramatsu et al demonstrated that YAP1 exhibited oncogenic properties in esophageal squamous-cell carcinoma ${ }^{18}$ and that patients with tumors overexpressing YAP1 had worse overall survival rates than those with nonoverexpressing tumors. Moreover, YAP1 positivity is independently associated with worse outcome in EC in multivariate analysis, indicating that it is a potential therapeutic target. ${ }^{18}$ Collectively, the above findings led us to hypothesize that targeted inactivation of YAP1 could suppress EC. However, there are few reports of the effect of YAP1 suppression in EC. 
A

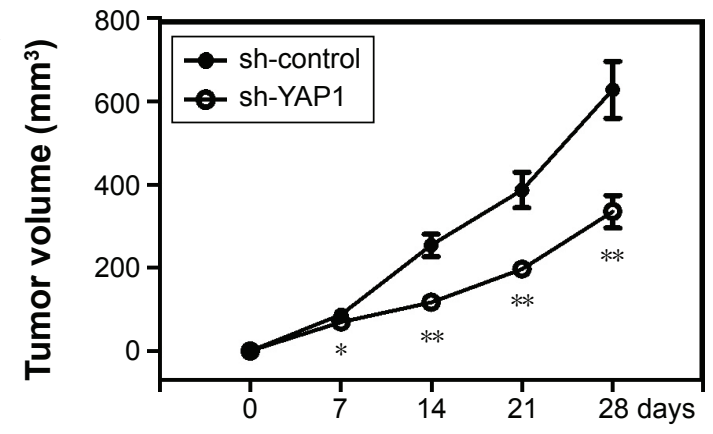

B

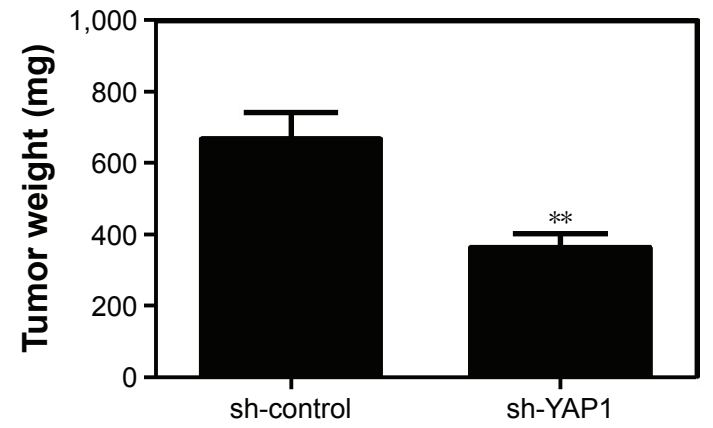

C

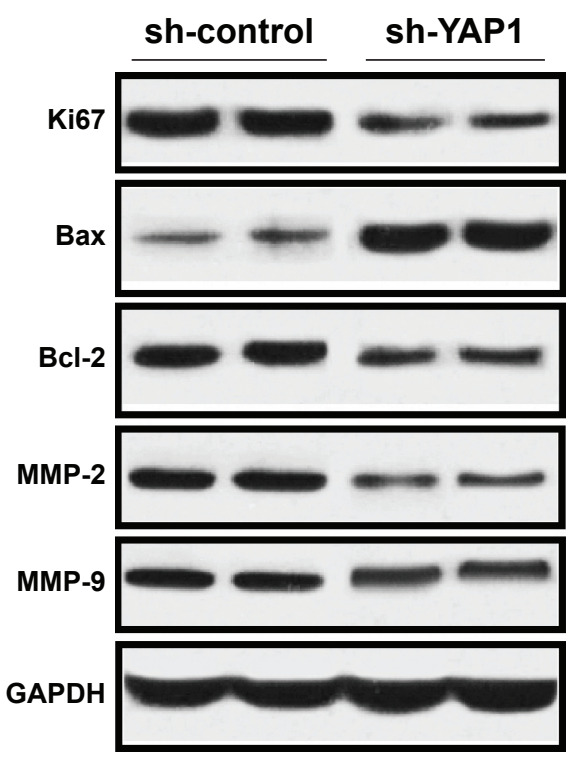

Figure 5 YAPI knockdown suppressed the growth of esophageal tumors in vivo.

Notes: (A) In the YAPI-knockdown group (sh-YAPI), the tumor volume was significantly decreased compared to that in the control group. (B) After 4 weeks, the tumor weight was significantly decreased in the YAPI-knockdown group compared to that in the control group. (C) YAPI knockdown decreased expression of Ki67, Bax, MMP-2, and MMP-9 and increased expression of $\mathrm{Bcl}-2$ compared to controls. $* P<0.05$ and $* * P<0.01$.

Abbreviation: sh-YAPI, short-hairpin RNA expression vector targeting YAPI.

In this study, we observed that YAP1 mRNA and protein were overexpressed in tumor samples from primary EC cases and EC cell lines and that YAP1 expression was significantly correlated with metastasis and EC tumor stage by retrospective analysis of clinical data. These data are consistent with those of Muramatsu et al. ${ }^{18}$

Tumorigenesis is a complex process and is accompanied by a series of changes, including changes in cell morphology, proliferation, apoptosis, and invasion. ${ }^{34}$ In our study, we investigated the effects of YAP1 knockdown on cell proliferation, apoptosis, and invasion in EC cell lines and found that they result in repression of cell proliferation and invasion and promote apoptosis. Similar results have been widely reported. Specifically, Diep et al reported that downregulation of YAP1 protein expression reduced cell proliferation and clonogenicity of pancreatic cancer cells; ${ }^{14}$ Muramatsu et al reported that YAP1 depletion repressed cell proliferation, mainly in the G0-G1 phase, and induced an increase in CDKN1A (p21) and a decrease in BIRC5 (Survivin) transcription in esophageal squamous-cell carcinoma; ${ }^{18}$ Overholtzer et al observed that the overexpression of YAP1 promoted growth factor-independent proliferation and anchorage-independent growth in the nontransformed mammary epithelial cell line MCF $10 A ;{ }^{19}$ Lamar et al reported that YAP1 promoted transformation, proliferation, migration, and invasion through its TEAD-interaction domain; ${ }^{6}$ and Menzel et al found that YAP1 promoted invasion and metastasis in melanoma. ${ }^{35} \mathrm{In}$ addition, a number of studies have demonstrated that the overexpression of YAP1 causes apoptosis in several tumors, including human esophageal squamous-cell carcinoma, hematological cancers, and prostate carcinoma. ${ }^{36-38}$

We also performed animal experiments to investigate the effect of YAP1 knockdown on the growth of esophageal tumors in vivo. Zender et al reported that YAP1 cooperated with the Myc oncogene to promote tumor growth in nude mice, ${ }^{15}$ and transgenic mice with liver-targeted YAP1 overexpression showed a significant increase in liver size, which eventually resulted in hepatic tumors. ${ }^{7,20}$ Consistent with these findings, our data indicate that YAP1 knockdown suppresses the growth of esophageal tumors in vivo.

\section{Conclusion}

In summary, upregulation of YAP1 mRNA and protein are frequently detected in EC, and YAP1 expression was significantly correlated with metastasis and EC tumor stage. YAP1 knockdown represses cell proliferation and invasion and promotes apoptosis in EC cell lines. In addition, YAP1 knockdown suppresses the growth of esophageal tumors in vivo in a mouse model. Owing to the limited number of EC samples and cell types available for this study, a further 
more comprehensive study will be required to investigate the potential role of YAP1 in the pathogenesis of EC. YAP1 knockdown could be explored for targeted gene therapy treatment for EC in the future.

\section{Disclosure}

The authors report no conflicts of interest in this work.

\section{References}

1. Siegel RL, Miller KD, Jemal A. Cancer statistics, 2015. CA Cancer J Clin. 2015;65(1):5-29.

2. Jiang Y, Chen S, Zhu B. Prognostic factors and family history for survival of esophageal squamous cell carcinoma patients after surgery. Ann Thorac Surg. 2010;90(3):908-913.

3. Yue D, Zhang Z, Li J, et al. Transforming growth factor-beta1 promotes the migration and invasion of sphere-forming stem-like cell subpopulations in esophageal cancer. Exp Cell Res. 2015;336(1):141-149.

4. Aminian A, Panahi N, Mirsharifi R, et al. Predictors and outcome of cervical anastomotic leakage after esophageal cancer surgery. $J$ Cancer Res Ther. 2011;7(4):448.

5. Ginn SL, Alexander IE, Edelstein ML, Abedi MR, Wixon J. Gene therapy clinical trials worldwide to 2012 - an update. J Gene Med. 2013; 15(2):65-77.

6. Lamar JM, Stern P, Liu H, Schindler JW, Jiang ZG, Hynes RO. The Hippo pathway target, YAP, promotes metastasis through its TEAD-interaction domain. Proc Natl Acad Sci U S A. 2012;109(37): E2441-E2450.

7. Zhao B, Wei X, Li W, et al. Inactivation of YAP oncoprotein by the Hippo pathway is involved in cell contact inhibition and tissue growth control. Genes Dev. 2007;21(21):2747-2761.

8. Kim NG, Koh E, Chen X, Gumbiner BM. E-cadherin mediates contact inhibition of proliferation through Hippo signaling-pathway components. Proc Natl Acad Sci U S A. 2011;108(29):11930-11935.

9. Zhao B, Li L, Wang L, Wang CY, Yu J, Guan KL. Cell detachment activates the Hippo pathway via cytoskeleton reorganization to induce anoikis. Genes Dev. 2012;26(1):54-68.

10. Wada K, Itoga K, Okano T, Yonemura S, Sasaki H. Hippo pathway regulation by cell morphology and stress fibers. Development. 2011; 138(18):3907-3914.

11. Dupont S, Morsut L, Aragona M, et al. Role of YAP/TAZ in mechanotransduction. Nature. 2011;474(7350):179-183.

12. Edgar BA. From cell structure to transcription: Hippo forges a new path. Cell. 2006;124(2):267-273.

13. Harvey K, Tapon N. The Salvador-Warts-Hippo pathway-an emerging tumour-suppressor network. Nat Rev Cancer. 2007;7(3):182-191.

14. Diep CH, Zucker KM, Hostetter G, et al. Down-regulation of Yes Associated Protein 1 expression reduces cell proliferation and clonogenicity of pancreatic cancer cells. PLoS One. 2012;7(3):e32783.

15. Zender L, Spector MS, Xue W, et al. Identification and validation of oncogenes in liver cancer using an integrative oncogenomic approach. Cell. 2006;125(7):1253-1267.

16. Fernandez-L A, Northcott PA, Dalton J, et al. YAP1 is amplified and up-regulated in hedgehog-associated medulloblastomas and mediates Sonic hedgehog-driven neural precursor proliferation. Genes Dev. 2009;23(23):2729-2741.

17. Snijders AM, Schmidt BL, Fridlyand J, et al. Rare amplicons implicate frequent deregulation of cell fate specification pathways in oral squamous cell carcinoma. Oncogene. 2005;24(26):4232-4242.

18. Muramatsu T, Imoto I, Matsui T, et al. YAP is a candidate oncogene for esophageal squamous-cell carcinoma. Carcinogenesis. 2010;32(3): 389-398.
19. Overholtzer M, Zhang J, Smolen GA, et al. Transforming properties of YAP, a candidate oncogene on the chromosome 11q22 amplicon. Proc Natl Acad Sci U S A. 2006;103(33):12405-12410.

20. Camargo FD, Gokhale S, Johnnidis JB, et al. YAP1 increases organ size and expands undifferentiated progenitor cells. Curr Biol. 2007;17(23): 2054-2060.

21. Strano S, Monti O, Pediconi N, et al. The transcriptional coactivator Yes-associated protein drives p 73 gene-target specificity in response to DNA damage. Mol Cell. 2005;18(4):447-459.

22. Basu S, Totty NF, Irwin MS, Sudol M, Downward J. Akt phosphorylates the Yes-associated protein, YAP, to induce interaction with 14-3-3 and attenuation of p73-mediated apoptosis. Mol Cell. 2003; 11(1):11-23.

23. Danovi S, Rossi M, Gudmundsdottir K, Yuan M, Melino G, Basu S. Yes-associated protein (YAP) is a critical mediator of c-Jun-dependent apoptosis. Cell Death Differ. 2008;15(1):217-219.

24. Yuan M, Tomlinson V, Lara R, et al. Yes-associated protein (YAP) functions as a tumor suppressor in breast. Cell Death Differ. 2008;15(11): 1752-1759.

25. Bertini E, Oka T, Sudol M, Strano S, Blandino G. YAP: at the crossroad between transformation and tumor suppression. Cell Cycle. 2009; 8(1):49-57.

26. Zhu X, Li Y, Shen H, et al. miR-137 inhibits the proliferation of lung cancer cells by targeting Cdc42 and Cdk6. FEBS Lett. 2013;587(1): 73-81.

27. Hao Y, Chun A, Cheung K, Rashidi B, Yang X. Tumor suppressor LATS1 is a negative regulator of oncogene YAP. J Biol Chem. 2008;283(9): $5496-5509$

28. Zhang J, Smolen GA, Haber DA. Negative regulation of YAP by LATS1 underscores evolutionary conservation of the Drosophila Hippo pathway. Cancer Res. 2008;68(8):2789-2794.

29. Liu AM, Xu MZ, Chen J, Poon RT, Luk JM. Targeting YAP and Hippo signaling pathway in liver cancer. Expert Opin Ther Targets. 2010; 14(8):855-868.

30. Pan D. The hippo signaling pathway in development and cancer. Dev Cell. 2010;19(4):491-505.

31. Wang Y, Dong Q, Zhang Q, Li Z, Wang E, Qiu X. Overexpression of yes-associated protein contributes to progression and poor prognosis of non-small-cell lung cancer. Cancer Sci. 2010;101(5):1279-1285.

32. Kang W, Tong JH, Chan AW, et al. Yes-associated protein 1 exhibits oncogenic property in gastric cancer and its nuclear accumulation associates with poor prognosis. Clin Cancer Res. 2011;17(8):2130-2139.

33. Zhang X, George J, Deb S, et al; AOCS Study group. The Hippo pathway transcriptional co-activator, YAP, is an ovarian cancer oncogene. Oncogene. 2011;30(25):2810-2822.

34. Birchmeier C, Birchmeier W, Gherardi E, Vande Woude GF. Met, metastasis, motility and more. Nat Rev Mol Cell Biol. 2003;4(12):915-925.

35. Menzel M, Meckbach D, Weide B, et al. In melanoma, Hippo signaling is affected by copy number alterations and YAP1 overexpression impairs patient survival. Pigment Cell Melanoma Res. 2014;27(4):671-673.

36. Imanaka Y, Tsuchiya S, Sato F, Shimada Y, Shimizu K, Tsujimoto G. MicroRNA-141 confers resistance to cisplatin-induced apoptosis by targeting YAP1 in human esophageal squamous cell carcinoma. J Hum Genet. 2011;56(4):270-276.

37. Cottini F, Hideshima T, Xu C, et al. Rescue of Hippo coactivator YAP1 triggers DNA damage-induced apoptosis in hematological cancers Nat Med. 2014;20(6):599-606.

38. Zagurovskaya M, Shareef M, Das A, et al. EGR-1 forms a complex with YAP-1 and upregulates Bax expression in irradiated prostate carcinoma cells. Oncogene. 2009;28(8):1121-1131. 


\section{Publish your work in this journal}

OncoTargets and Therapy is an international, peer-reviewed, open access journal focusing on the pathological basis of all cancers, potential targets for therapy and treatment protocols employed to improve the management of cancer patients. The journal also focuses on the impact of management programs and new therapeutic agents and protocols on

patient perspectives such as quality of life, adherence and satisfaction. The manuscript management system is completely online and includes a very quick and fair peer-review system, which is all easy to use. Visit http://www.dovepress.com/testimonials.php to read real quotes from published authors.

Submit your manuscript here: http://www.dovepress.com/oncotargets-and-therapy-journal 